\title{
THE RADIUS OF CLOSE-TO-CONVEXITY OF FUNCTIONS OF BOUNDED BOUNDARY ROTATION
}

\author{
H. B. COONCE${ }^{1}$ AND M. R. ZIEGLER
}

\begin{abstract}
An analytic function whose boundary rotation is bounded by $k \pi(k \geqq 2)$ is shown to map a disc of radius $r_{k}$ onto a close-to-convex domain, where $r_{k}$ is the solution of a transcendental equation when $k>4$ and $r_{k}=1$ when $2 \leqq k \leqq 4$. The above value of $r_{k}$ is shown to be the best possible for each $k$ and an asymptotic expression for $r_{k}$ is obtained.
\end{abstract}

Let $V_{k}(k \geqq 2)$ denote the class of functions $f(z)$ which are analytic in the unit disc $E=\{z:|z|<1\}$, normalized by $f(0)=0$ and $f^{\prime}(0)=1$, have nonvanishing derivatives in $E$, and map $E$ onto a domain which has boundary rotation at most $k \pi$. If $k=2$, then $V_{k}$ is precisely the set of univalent functions which map $E$ onto a convex domain. If $2<k \leqq 4$, then $V_{k}$ is a subset of the functions which map $E$ onto a close-to-convex domain ([1], [6]). Finally, if $k>4$, then functions in $V_{k}$ need not be close-to-convex or even univalent. In this paper we determine the radius of close-to-convexity of $V_{k}$ for each $k$, i.e. the radius of the largest disc centered at the origin which is mapped onto a close-to-convex domain by all $f$ in $V_{k}$. The techniques used are similar to those used by Krzyż in determining the radius of closeto-convexity of the class of univalent functions [2]. Some related problems were posed by M. O. Reade [5].

THEOREM 1. If $k>4$, the radius of close-to-concexity of $V_{k}$ is the unique root of the equation

$$
2 \cot ^{-1} w-k \cot ^{-1}(k w / 2)=-\pi
$$

in the interval $\left(R_{k}, 1\right)$ where $R_{k}$ is the radius of concexity of $V_{k}$ and $w=$ $\left(1-r^{2}\right)\left[k^{2} r^{2}-\left(1+r^{2}\right)^{2}\right]^{-1 / 2}$, while if $2 \leqq k \leqq 4$, the radius of close-to-concexity is 1 .

Proof. Kaplan [1] has shown that a necessary and sufficient condition for a function $f^{\prime}(z)$, regular in $E$ and satisfying $f^{\prime}(z) \neq 0$ : to map $|z|=r$ onto

Presented to the Society, January 18, 1972; received by the editors October 14, 1971 and, in revised form, January 3, 1972.

AMS 1970 subject classifications. Primary 30A32.

Key nords and phrases. Bounded boundary rotation, radius of close-to-convexity.

${ }^{1}$ This research was supported in part by a Mankato State College Faculty Research Council Grant. 
a close-to-convex curve is that

$$
\arg \left[z_{2} f^{\prime}\left(z_{2}\right)\right]-\arg \left[z_{1} f^{\prime}\left(z_{1}\right)\right] \geqq-\pi
$$

for all $z_{1}$ and $z_{2}$ with $\left|z_{1}\right|=r$ and $z_{2}=z_{1} e^{i \theta}, 0<\theta<2 \pi$. The radius of closeto-convexity of $V_{k}$ is the largest value of $r$ for which (2) holds for all $f(z)$ in $V_{k}$. The radius of convexity $R_{k}$ of $V_{k}$ is the smallest positive root of the equation $1-k r+r^{2}=0 ; R_{2}=1$ and $R_{k}<1$ when $k>2$ [3]. Clearly the radius of close-to-convexity is larger than $R_{k}$ when $k>2$ and equal to $R_{k}$ when $k=2$, hence we assume throughout the remainder of this work that $r>R_{k}$ and $k>2$.

Define

$$
\Delta(r, \theta)=\inf _{f \in V_{k}^{\prime}} \arg \left[z_{2} f^{\prime}\left(z_{2}\right) / z_{1} f^{\prime}\left(z_{1}\right)\right]
$$

where $z_{1}$ and $z_{2}$ are defined as above and the argument is chosen to vary continuously from an initial value of zero. Let $\zeta=\left(z-z_{1}\right) /\left(1-\bar{z}_{1} z\right)$ and $\zeta_{0}=\left(z_{2}-z_{1}\right) /\left(1-\bar{z}_{1} z_{2}\right)$ and define $g(\zeta)$ by

$$
g(\zeta)=\left[f\left(\left\{\zeta+z_{1}\right\} /\left\{1+\bar{z}_{1} \zeta\right\}\right)-f\left(z_{1}\right)\right] / f^{\prime}\left(z_{1}\right)\left(1-\left|z_{1}\right|^{2}\right) .
$$

Robertson has shown that $g(z)$ is in $V_{k}$ whenever $f(z)$ is in $V_{k}$ [7]. Evaluating $g^{\prime}\left(\zeta_{0}\right)$ directly yields

$$
g^{\prime}\left(\zeta_{0}\right)=f^{\prime}\left(z_{2}\right)\left(1-z_{1} z_{2}\right)^{2} / f^{\prime}\left(z_{1}\right)\left(1-\left|z_{1}\right|^{2}\right)^{2}
$$

hence we have $\Delta(r, \theta)=\arg \left[\left(z_{2} / z_{1}\right)\left(1-\bar{z}_{1} z_{2}\right)^{-2}\right]+\inf \arg _{g_{\in} V_{i}}\left[g^{\prime}\left(\zeta_{0}\right)\right]$. Now

and

$$
\begin{gathered}
\arg \left[\left(z_{2} / z_{1}\right)\left(1-z_{1} z_{2}\right)^{-2}\right]=2 \cot ^{-1}\left[\left(1-r^{2}\right) \cot (\theta / 2) /\left(1+r^{2}\right)\right], \\
\left|\zeta_{0}\right|=r\left[2(1-\cos \theta) /\left(1-2 r^{2} \cos \theta+r^{4}\right)\right]^{1 / 2}
\end{gathered}
$$

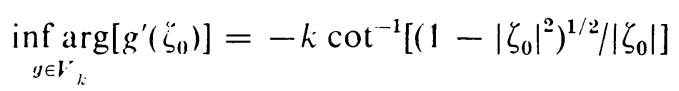

thus a brief calculation shows

$$
\begin{aligned}
\Delta(r, \theta)= & 2 \cot ^{-1}\left[\left(1-r^{2}\right) \cot (\theta / 2) /\left(1+r^{2}\right)\right] \\
& -k \cot ^{-1}\left[\left(1-r^{2}\right) / r\{2(1-\cos \theta)\}^{1 / 2}\right] .
\end{aligned}
$$

Furthermore, this estimate is sharp since, for a fixed $z_{1}$ and $z_{2}$, if $g(\zeta)$ is the function which gives equality in (4) and $f(z)$ is defined by

$$
f(z)=\left[g\left(\left\{z-z_{1}\right\} /\left\{1-\bar{z}_{1} z\right\}\right)-g\left(-z_{1}\right)\right] / g^{\prime}\left(-z_{1}\right)\left(1-\left|z_{1}\right|^{2}\right),
$$

then equality occurs in (3) for this choice of $f(z)$. Let $\Delta(r)=\inf \Delta(r, \theta)$ $(0<\theta<2 \pi)$. Differentiating (5) with respect to $\theta$ we obtain

$$
\partial \Delta(r, \theta) / \partial \theta=\left[1+r^{2}-k r \cos (\theta / 2)\right]\left(1-r^{2}\right) /\left(1-2 r^{2} \cos \theta+r^{4}\right) ;
$$


hence $\Delta(r, \theta)$ assumes its minimum value for a fixed $r$ when $\theta=\theta_{0}$ where $\cos \left(\theta_{0} / 2\right)=\left(1+r^{2}\right) / k r$. The existence of $\theta_{0}$ is assured by the fact that for $r>R_{k},\left(1+r^{2}\right) / k r<1$. Substituting in (5), we have

$$
\Delta(r)=2 \cot ^{-1} w^{\prime}-k \cot ^{-1}(k w / 2)
$$

where $w=\left(1-r^{2}\right)\left[k^{2} r^{2}-\left(1+r^{2}\right)^{2}\right]^{-1 / 2}$. It is evident that $\Delta(r)$ is a decreasing function of $r$, hence $\Delta(r) \geqq \Delta(1)=\pi(2-k) / 2$. For $k \leqq 4, \Delta(1) \geqq-\pi$ and the radius of close-to-convexity is 1 , while for $k>4, \Delta(1)<-\pi$ and $\Delta\left(R_{k}\right)=0$; hence there exists a unique solution $r_{k}$ to the equation $\Delta(r)=-\pi, R_{k}<r<1$, and this solution is the radius of close-to-convexity.

Table 1 gives the approximate value of $r_{k}$ for various $k$. [The calculations were performed on a Univac 1106 by Mr. Michael Barnett of the Computer Science Center of Mankato State College.]

TABLE 1

\begin{tabular}{rlrcrc}
$k$ & \multicolumn{1}{c}{$r_{k}$} & $k$ & $r_{k}$ & $k$ & $r_{k}$ \\
4 & 1 & 9 & 0.34593 & 50 & 0.05952 \\
5 & 0.70388 & 10 & 0.30849 & 100 & 0.02973 \\
6 & 0.55362 & 20 & 0.14994 & 200 & 0.01486 \\
7 & 0.45961 & 30 & 0.09946 & 400 & 0.00743 \\
8 & 0.39431 & 40 & 0.07446 & 800 & 0.00371
\end{tabular}

THEOREM 2. $\lim _{k+\infty} k r_{k}=2.9716 \ldots=\alpha$ where $\alpha$ is the unique root of the equation

$$
\cot ^{-1}\left[\left(\alpha^{2}-1\right)^{-1 / 2}\right]-\left(\alpha^{2}-1\right)^{1 / 2}=-\pi / 2
$$

in the interval $[\pi / 2, \pi]$.

Proof. If $f(z)$ is in $V_{k}$, then (4) implies $\operatorname{Re}\left\{f^{\prime}(z)\right\}>0$ for $|z|<\pi / 2 k$. $\operatorname{Re}\left\{f^{\prime}(z)\right\}>0$ is a sufficient condition for close-to-convexity, hence $r_{k} \geqq \pi / 2 k$. An examination of the mapping properties of the function

$$
f_{0}(z)=(1 / k)\left\{[(1+z) /(1-z)]^{1,2}-1\right\}
$$

shows that the radius of univalence $\rho_{k}$ of $f_{0}(z)$ satisfies $\rho_{t:}=\csc (2 \pi / k)-$ $\cot (2 \pi / k)$. Since $\lim k \rho_{k}=\pi(k \rightarrow \infty)$, we have $\gamma_{0}=\lim$ sup $k r_{k} \leqq \pi(k \rightarrow \infty)$. If $\left\{k_{n}\right\}$ is any sequence such that $\lim k_{1 \prime} r_{k_{n}}=\%(n \rightarrow \infty)$, then it follows from (1) that $\alpha$ satisfies (7). However a differentiation of (7) shows the left-hand side to be a monotonic decreasing function and thus $\lim k r_{l} .(k \rightarrow \infty)$ must exist and is the unique root of (7). 


\section{REFERENCES}

1. W. Kaplan, Close-to-convex schlicht functions, Michigan Math. J. 1 (1952), 169185. MR 14, 966

2. J. Krzyż, The radius of close-to-convexity within the family of univalent functions, Bull. Acad. Polon. Sci. Sér. Sci. Math. Astronom. Phys. 10 (1962), 201-204. MR 26 \#6384.

3. V. Paatero, Über Gebiete von beschrankter Randdrehung, Ann. Acad. Sci. Fenn. Ser. A 37 (1933), 9.

4. B. Pinchuk, A variational method for functions of bounded boundary rotation, Trans. Amer. Math. Soc. 138 (1969), 107-113. MR 38 \#6042.

5. M. O. Reade, Ann. Polon. Math. 20 (1968), p. 317, problem 5.

6. A. Rényi, Some remarks on univalent functions, Bŭlgar. Akad. Nauk. Izv. Mat. Inst. 3 (1959), no. 2, 111-121. MR 22 \#773.

7. M. S. Robertson, Coefficients of functions with bounded boundary rotation, Canad. J. Math. 21 (1969), 1477-1482. MR 41 \#458.

Department of Mathematics, Mankato State College, Mankato, Minnesota 56001

Department of Mathematics, Marquette University, Milwaukee, Wisconsin 53233 